\title{
Cladistics
}

\section{Myth of the molecule: DNA barcodes for species cannot replace morphology for identification and classification}

\author{
Kipling W. Will ${ }^{\mathrm{a}, *}$ and Daniel Rubinoff ${ }^{\mathrm{b}}$ \\ ${ }^{a}$ University of California, Department Environmental Science, Policy and Management, Division of Insect Biology, Berkeley, CA 94720, USA \\ ${ }^{b}$ University of Hawaii, Department of Plant and Environmental Protection Sciences, 3050 Maile Way,
} 310 Gilmore Hall, Honolulu, HI 96822, USA

Accepted 8 December 2003

\begin{abstract}
So-called DNA barcodes have recently been proposed to answer the problem of specimen identification and to quantify global biodiversity. We show that this proposition is wanting in terms of rationale, methodology and interpretation of results. In addition to falling short of all its stated goals, the method abandons the benefits of morphological studies in favor of a limited molecular identification system that would ultimately impede our understanding of biodiversity.
\end{abstract}

(C) The Willi Hennig Society 2004.

"If the only tool you have is a hammer, you tend to see every problem as a nail.' Abraham Maslow

DNA barcodes for species-level identification may, at first glance, seem to represent an appropriate use of new technology to solve an old problem-identifying and classifying the world's biodiversity. Working toward this goal of understanding biodiversity is commendable, but we see serious flaws in the rationale, methodology, and interpretation of results involved in abandoning morphological studies in favor of a narrow and wholly molecular identification system, as suggested by Hebert et al. (2003a). Recently the concept of DNA taxonomy, of which the DNA barcode is one instance, has been hotly debated (Tautz et al., 2002, 2003; Lipscomb et al., 2003; Pennisi, 2003; Scotland et al., 2003a; Seberg et al., 2003) and the DNA barcode concept in general has been challenged (Scotland et al., 2003a; Sperling, 2003). Despite these significant challenges and existing literature suggesting that the method of DNA barcodes is unsound, claims that the technique has been validated are still being published (Hebert et al., 2003b; Stoeckle

\footnotetext{
*Corresponding author. E-mail addresses: kiplingw@nature.berkeley.edu (K.W. Will), rubinoff@hawaii.edu (D. Rubinoff)
}

et al., 2003; Stoeckle, 2003; Whitfield, 2003; including many references at the website). Herein we demonstrate, using the examples published by Hebert et al., that the methodology fails not only theoretically, but also on a practical level. Additionally, we develop arguments and synthesize previously published views (e.g. Scotland et al., 2003a; Sperling, 2003), specifically applying them to claims made by Hebert et al. (2003a). The intent of this paper is to clarify the practical and theoretical shortcomings that would result from the adoption of a DNA barcoding system to identify biodiversity.

\section{Discussion}

Obviously there are many more species-level taxa to be recognized and specimens to be identified, even by conservative estimates (Novotny et al., 2002), than the present or predicted levels of manpower can handle given the limitations of current technology. As a result, users of biological classifications are interested in streamlining procedures to arrive at a confidently applied, uniform taxonomic concept for organisms under study. Hebert et al. (2003a) presented methods that attempted to shortcut these identifications and respond to the dearth of taxonomists. 
While it is unclear what Hebert et al. meant by "critical" identification (p. 313), it seems likely, based on their presentation of results, that for them "critical" is equivalent to an accurate identification. Accuracy, in this case, that is wholly dependent on the existing taxonomy of the group. Unfortunately, to the dismay of people seeking an immediate panacea, the molecular identification of species is fraught with the same constraints and inconsistencies that plague morphological judgments of species boundaries. The exception is that most morphologically knowledgeable workers have a suite of complex morphological characters upon which to base their conclusions, rather than relying on part of a single gene.

In regard to the shortage of expertise to carry out identifications, Hebert et al. suggested that 15000 taxonomists would be needed for perpetuity "to identify life". This demonstrates a fundamental misunderstanding that may be rather widespread in biology. Taxonomy and systematics are not service industries for other fields of inquiry (Lipscomb et al., 2003), but rather represent viable scientific endeavors in their own right, with broad application to other fields of science and industry. Researchers in these fields are expected to propose taxonomic hypotheses and develop identification tools essential to the rest of biology, not to provide routine identifications. As an analogy, researchers in electron optics are not expected to do routine scanning electron microscopy work. Of course, many specimens are identified by experts in the process of their taxonomic research and in partnership with other biologists. Typically, this is a mutually beneficial arrangement. Our taxonomic concepts are, or should be, built on a constellation of data, which in part is often gathered by non-systematists during their investigations. Reciprocity in the system may slow progress, but the ultimate products are of greater value. Hebert et al.'s interpretation of the biodiversity/systematics problem and the solutions offered are a cause for concern. If a program such as they suggest were initiated at the expense of morphological systematics, we feel there would be serious negative consequences for accurate biodiversity assessments. The following response is intended to point out problems with Hebert et al.'s proposal at different levels from interpretation to data collection and analysis.

\section{Always room for error}

Despite claims that DNA barcoding methods work, it is clear that non-identical sequences may remain unidentifiable or may be unambiguously wrongly placed. A basic property of the barcode method is that there will always be internal attachment points that are ambiguous in terms of identification for any rooted cladogram. Examples of this can be seen in subtrees taken from Hebert et al. (their electronic appendix D, our Fig. 1; their appendix E, our Fig. 2). In the subtree Fig. 1 the number of non-informative attachment points at the generic level is 19 of 50 total possible positions at which a new "test" sequence could attach. Similarly in Fig. 2, the number of non-informative attachment points is 11 of 30 total for species-level and 8 of those are also uninformative at the generic level. The potential problem with non-informative attachment points is only avoided with certainty if the sequence is identical to one already included in the "profile". For example (Fig. 2), if non-identical sequence data from a second and third individual of Simyra henrici were included, and if they prove to form a paraphyletic group, being placed above and below the existing terminal, the term below would be unidentifiable and the term above would be positively attributed to the genus Acronicta. Neither result is acceptable. Similarly, if the original analysis had not included $A$. impressa and $A$. lepusculina, giving the resulting relationships ( $A$. dactylina $(S$. henrici $(A$. hasta + $A$. morula))), adding two unknowns that were in fact [1] A. impressa and [2] A. lepusculina, results in relationships (([1], A. dactylina ([2] (S. henrici (A. hasta + A. morula))). In these paraphyletic relationships it is impossible to recover any taxonomic information below the suprageneric level, not even genus membership.

It is very surprising that no test taxon included by Hebert et al. in any of the results they presented, grouped at any internal node (simulation studies may be needed to understand this phenomenon). However, one can clearly see that if the nematode NE 8 (their Fig. 1 phylum profile) or the coleopteran Coccinelidae (their fig. 2 ordinal profile) had been a "test" taxon and not a "profile" taxon, their placement in Phylum and Order, respectively, would have been impossible or incorrect, e.g. the lady-bird beetle would be a basal wasp!

Hebert et al. do tout their recovery of 18 "monophyletic pairs" of species from genera represented by two taxa in their analysis of 200 lepidopterans. This monophyly rate $(78 \%)$ drops to only 24 of $35(69 \%)$ when all genera with more than one representative are considered. This supposed "evidence for clustering of taxonomically allied species" strikes us as a failing rate of recovery at best. Relying on a system that fails $31 \%$ of the time to identify all of life leaves millions of specimens misidentified.

For species identification, when a species (or divergent population) is not already sequenced, the prospects are worse yet. Adding a novel Acronicta species to Fig. 2 is an empty exercise, as all possible attachment points are either non-informative or positively wrong.

To a large degree, the conflation of phylogenetic methods and phylogenies with phenetic distances and clustering creates serious problems for DNA barcoding. Hebert et al. deftly avoid the term "phylogeny" by using the term "profile", presumably to prevent an interpretation of their essentially phenetic results as phylogenetic 


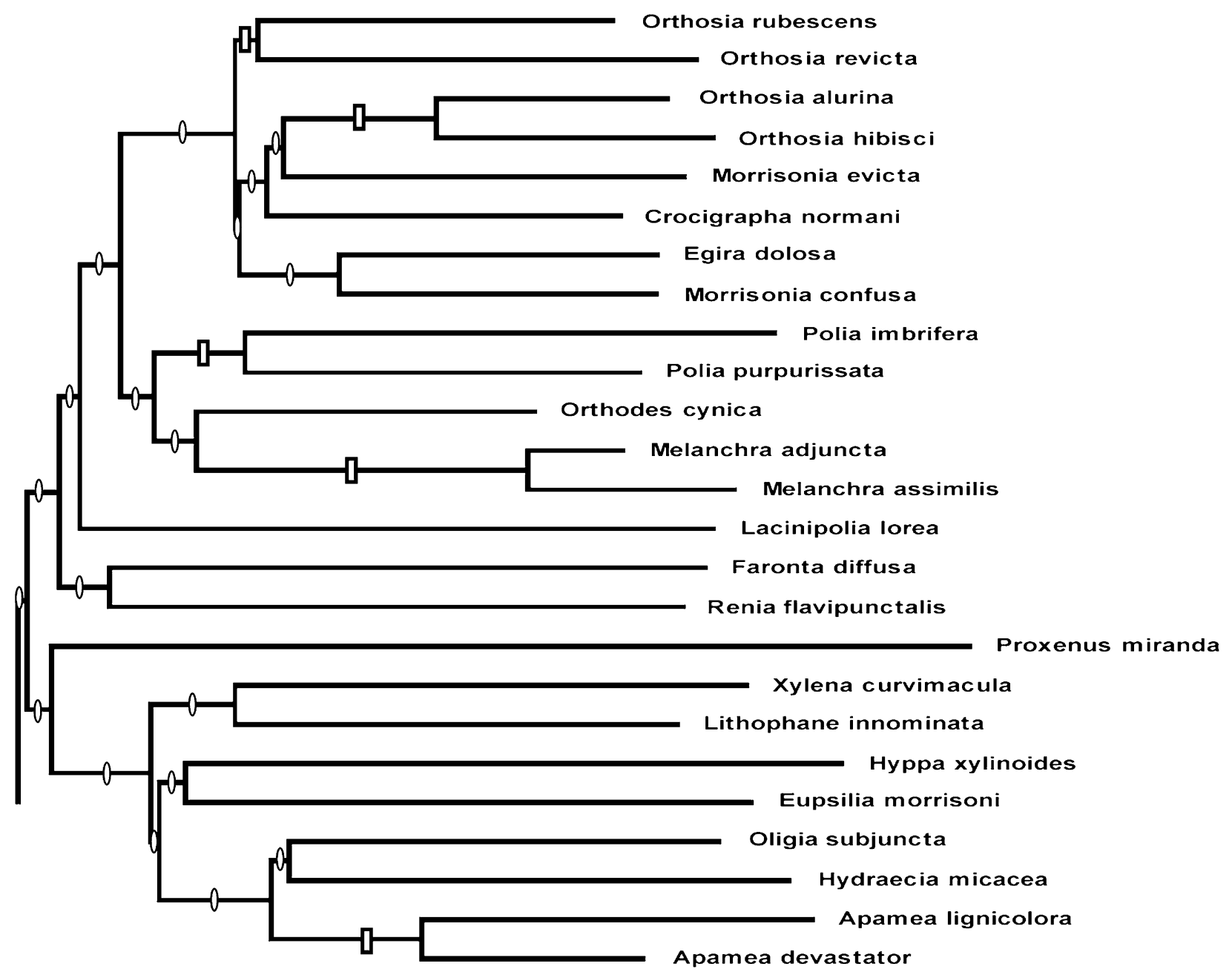

Fig. 1. Modified subtree from appendix D of Hebert et al. (2003a). Open ellipses indicate non-informative internal branches at the generic level and open rectangles indicate non-informative internal branches at the species level.

relationships. A critical inspection of any of their tree diagrams reveals that these are implausible phylogenies of the included groups, which do not concur with current or previous hypotheses. Nevertheless, Hebert et al. use chimeric terminology, e.g. monophyletic and the undefined "cohesive group". 1 They do justify their choice to use neighbor-joining (NJ) methods by reference to the purported "strong track record" of the method in recovering phylogenies (their citation of Kumar and Gadagkar, 2000) and use Poisson correction to "reduce the impacts of homoplasy". In fact, what they have produced is a series of extremely poor phylogenies that are symptomatic of the underlying confusion of "profiles" and phylogenies. Ignoring the failed phylogenetic reconstructions, one must then focus

\footnotetext{
${ }^{1} \mathrm{We}$ assume a cohesive group in their sense is in fact a convex group. A term introduced by pheneticists (Estabrook, 1978, 1986) that includes both monophyletic and paraphyletic groups.
}

on only phenetic clustering such that " 'test' taxa [are] assigned to the proper phylum/order" or "grouped most closely with the single representative" for a species. Exactly how one tells a taxon has been "assigned" to one order or another is not explained. This confusion of phenetics and phylogenetics further obscures the implications from the results of this study.

\section{Distance matrix and species identifications}

As Hebert et al. point out (p. 314), mtDNA sequence varies and its rate of evolution is inconsistent within and between species. Therefore, there is no standard level of divergence that can delineate species boundaries - even within families of Lepidoptera. Thus, using the methodology in Hebert et al. a researcher who obtains DNA data that is not an exact match for a previously sequenced and identified species has virtually no way of knowing exactly what he or she has got, 


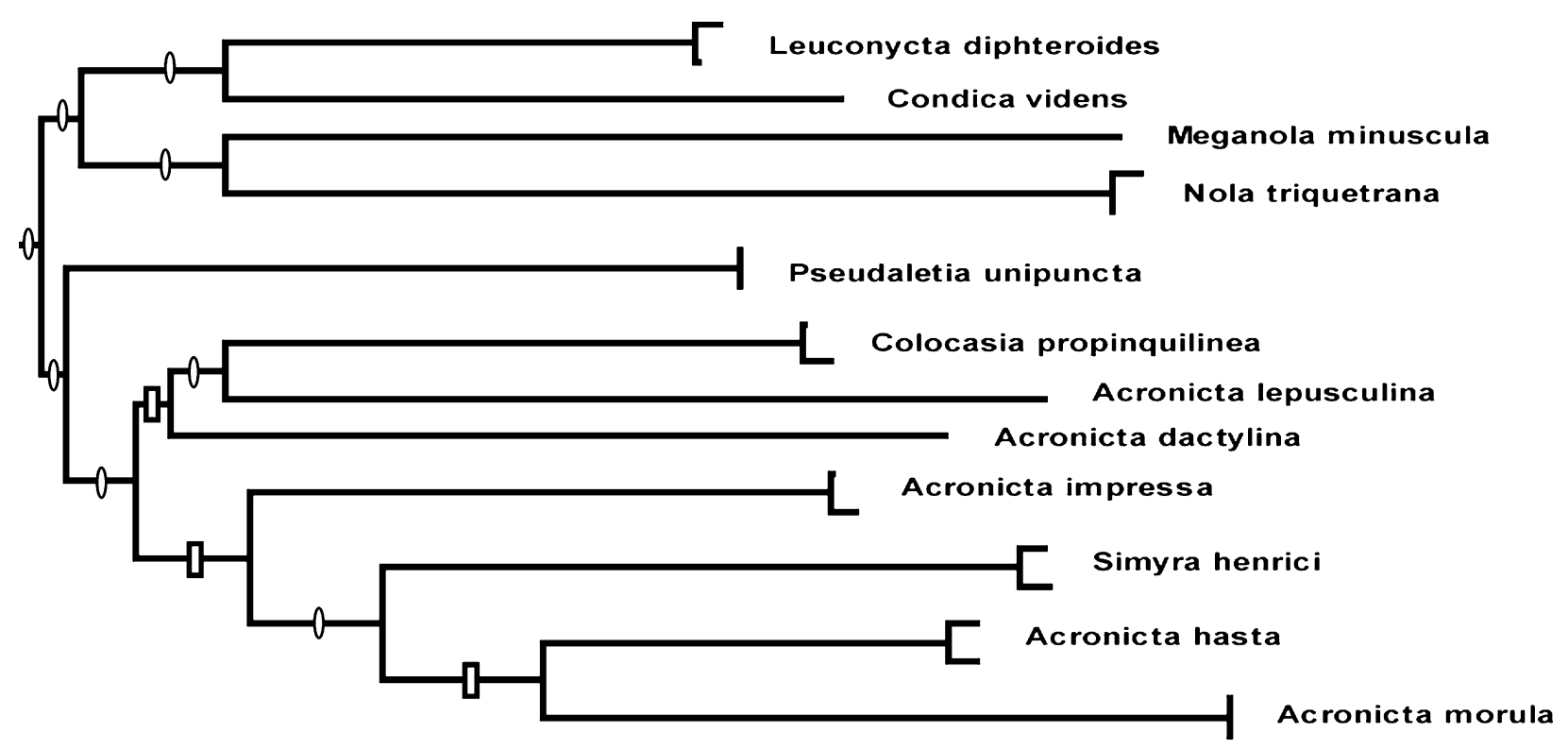

Fig. 2. Modified subtree from appendix E of Hebert et al. (2003a). Open ellipses indicate non-informative internal branches at the generic level and open rectangles indicate non-informative internal branches at the species level.

since there is no standardized distance for all species. Hebert et al. argue that the "hierarchical clusters of pseudogroups" that would result from a cladistic analysis would not be as useful as the Euclidean space they use to map their taxa. In fact, their phenetic methodology is a profound weakness of their presentation. A cladistic method would, at least, offer hypotheses regarding levels of relatedness, even if a new taxon was not an exact match for a previously identified specimen. Their distance matrix offers no additional information. Nevertheless, whether cladistic or phenetic, DNA barcode methods fail intrinsically, since any "new" species introduced to their distance matrix must fit almost exactly on the coordinates of a previously sequenced species (which must have been identified by a taxonomist!). While their "test" species fell closest to their conspecifics in three of the eight tests $(37.5 \%)$, the test species was not an exact match. Because the parameters of the spatial region constituting a species are undefined in such a matrix (and so is simply an extension of the "species" debate (Wilson, 1999; Wheeler and Meier, 2000)), a "near miss" may or may not be a conspecific. Thus, a near miss leaves the researcher back at the "mercy" of a taxonomist to confirm the identification. Even at the genus level, if a new taxon falls within the "radius" of more than one genus (Fig. 3, modified from Fig. 4 of Hebert et al.) then sequence data under Hebert et al.'s analysis yields no information about the "new" taxon, and it must be physically identified by a taxonomist to determine not only species, but genus as well. To illustrate this point, if the distance between Sphinx gordius and Sphinx canadensis were used to identify a radius of Euclidean space in which other members of the genus Sphinx should fall (a reasonable assumption that one or the other species might be near the "center" of Sphinx genus coordinates), this radius would include four other genera besides Sphinx (Fig. 3). In the case presented by Hebert et al. the problem would be even greater if Smerinthus were used. Even if the congeneric taxa just mentioned represented (through an unhappy coincidence) the extremes of congeneric space for their respective genera, i.e. an actual generic radius of half of what is currently apparent in Fig. 3, such a graphic would still fail to identify most Sphingid genera as they are currently described. In this situation a taxon introduced to the matrix which did not fit nearly exactly on a pre-identified point would give no information below the family level.

When testing the accuracy of their distance matrix for species level identifications, they used single representatives. This single representative species approach on a distance matrix is essentially typological, allowing for little - and unspecified - variation between individuals which may or may not be conspecific. The concept that there is one "true" point in space that represents and defines a species is generally regarded as passé (Wilson, 1999; Wheeler and Meier, 2000) and does not represent the variability commonly seen within species. For well-differentiated taxa such an approach might function when the distances between species are large and unfettered by taxa or individuals with intermediate coordinates. However, in such situations morphological identifications are typically easi- 


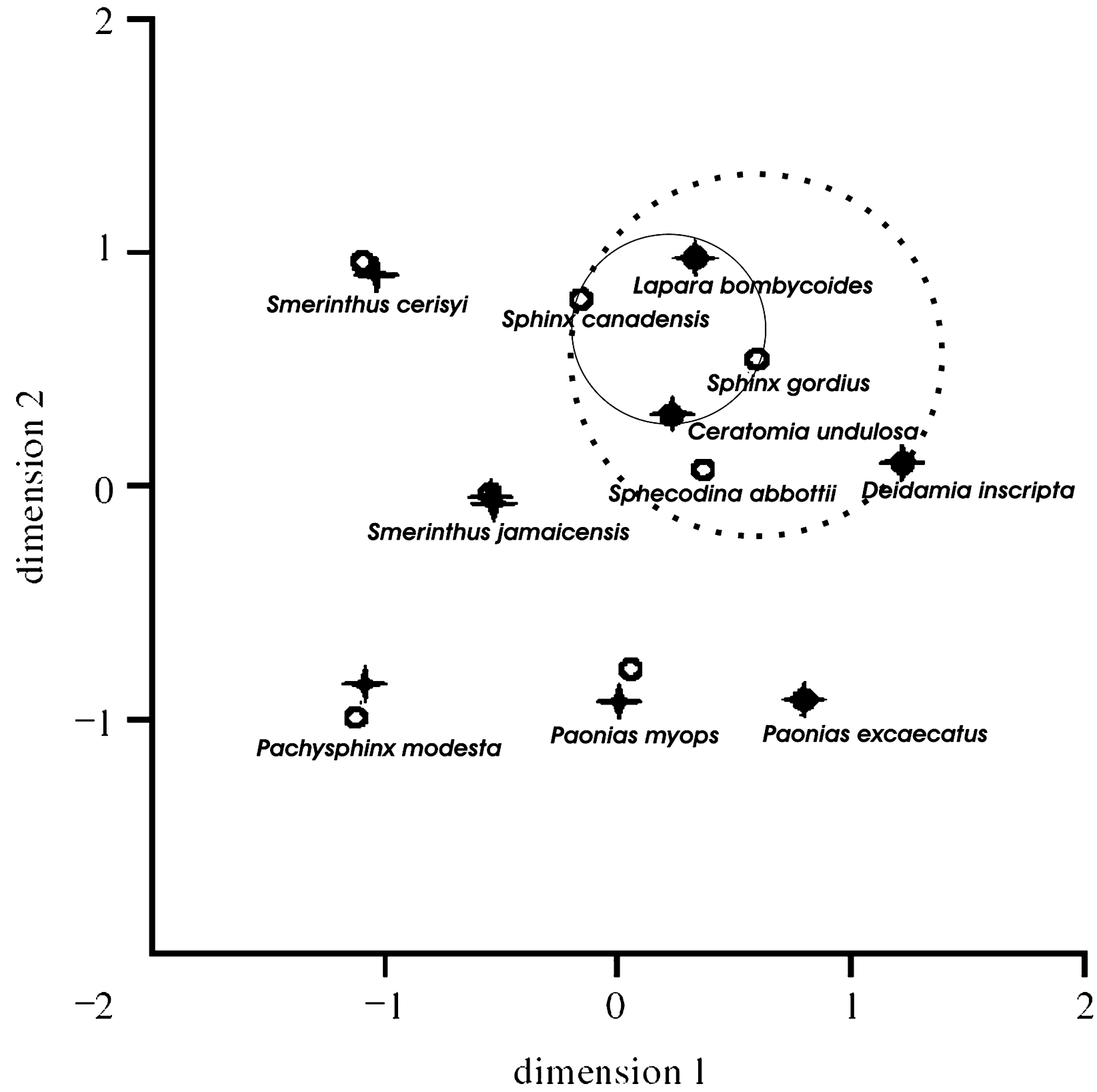

Fig. 3. Multi-dimensional scaling of Euclidean distances from 11 species of Sphingidae adapted from Hebert et al. (2003a). The solid circle represents a conservative estimate of Euclidean space in which members of the genus Sphinx might be expected to fall if $S$. gordius and $S$. canadensis represented the extreme pair-wise distance for the genus. The dotted circle represents a Euclidean space for the genus Sphinx if $S$. gordius were centrally located in the generic space of Sphinx and S. canadensis represented the most distant member of the genus. If the space were not circular, then the representation of generic space occupied by a genus is unpredictable. Note that both circles contain taxa not in the genus Sphinx. Species plots from original, hypothetical circular bounds representing the space occupied by the Sphinx genus have been added.

est to perform, and any advantage sequencing offers is negligible. When species are closely related, dispersed irregularly across the distance matrix or for any other reason poorly differentiated, the determination of affiliations for "new species" that are not exact hits is ambiguous. Given the arbitrary nature of the methods and results given by Hebert et al. (2003a), attempting to use their approach to identify the world's biodiversity verges on ludicrous.

\section{Limitations}

A recognized manpower shortage is coupled by Hebert et al. to what they consider four limitations of 
using morphology for identification. We will briefly discuss each of these in turn.

"phenotypic plasticity and genetic variability [...] can lead to incorrect identification"

Phenotypic plasticity in many cases is well understood and certainly well recognized in morphological systems. In fact, we have over 200 years of observations that make us aware of this issue, and it is this background that has allowed biologists to repeatedly correct taxondefinitions when non-heritable variation is recognized. The larger issue affected by phenotypic plasticity is species (or other taxonomic group) definition. This problem should not be confused with taxonomic identification, i.e. group membership. Once the operational species boundaries are determined, identification either results in the inclusion of the organism under study in a group or not. If the individual does not have the diagnostic combination of characters of a named taxon then re-definition is necessary, i.e. placement in a new or different group, or expansion of the existing taxon definition. For those groups that have a complete overlap of morphological characters in some individuals, the discriminating criteria, which should exist if the group is to be recognized at all, must be used (e.g. behaviors or pheromone recognition). If those features are not available, then molecular data may be the best or only answer for some limited set of taxa and/or a given taxonomic level. The broad application of DNA identification across life based on this "limitation" is, however, the tail wagging the dog.

Hebert et al. do not explain their use of "genetic variability" in the context of a limitation to morphological identification. Given that within-gene, base variation is not problematic for morphological identification per se, we assume that they mean that there is a mismatch between phenotypic variation and genetic variation. One possible interpretation of their use of genetic variability is in reference to underlying mechanisms that may differ for a similar looking anatomical feature in two taxa, potentially resulting in homoplasy. Thus, the feature is not assumed to be a reliable indicator of identity. This presumes a one-to-one relationship between the genetic pathway underlying the expressed characteristic and the structure observed. Clearly this is an oversimplification and there is ample evidence to show that the same trait may be produced by more than one means within a group (Wagner, 1994; Schlichting and Pigliucci, 1998).

It is well known that multiple gene families have members with different histories, i.e. gene vs. species trees (Avise, 2000), and that gene elements may move from one part of the genome to another giving the impression that the structure is non-homologous. Regardless of how the elytra of a beetle or spinneret of a spider is produced during development, organisms with those features are classified as beetles or spiders. A phylogenetic study, based on a broader selection of characters (including developmental characters, if available), would be required to show that all elytra are not homologs. This, like species identification vs. delimitation, is a question fundamental to phylogenetic research, but background information for taxonomic identifications.

Alternatively, they may mean that genetic variation may be greater than morphological variation, or the reverse. The first is exactly equivalent to "cryptic taxa" discussed below and the latter exactly equivalent to "phenotypic plasticity" above. Both assume that the small gene fragment used bears the correct marks of history for the specific group and age of the lineage. However, real world experience is to the contrary.

\footnotetext{
"Second, this approach overlooks morphologically cryptic taxa..."
}

If Hebert et al. mean taxa that are not recognizably different in gross morphology (and not just hard to differentiate because the characteristics are not obvious to the eye) but are composed of genetically and/or reproductively isolated units, then this is certainly a special case in biology. By definition these units must be discovered and separated by extraordinary means. Usually this is through behavioral, morphometric, biochemical or molecular data and done by an expert on the taxon. For these taxa, sequence data are likely to be the most effective means for discovery and differentiation. This, like purported limitations due to variation above, is only a problem for selected taxa and is realized only after study and phylogenetic analyses show that a unit exists, which has not been otherwise exposed, and is worthy of recognition. In terms of routine identifications for all life, which the authors claim to be addressing, justification based on this limitation is wanting outside of groups like bacteria, or other micro-organisms where DNA is the best or only option.

Hebert et al. then note that keys are specific to a single semaphoront. ${ }^{2}$ We agree that the paucity of suitable keys is problematic. However, there still remains a huge gap between the number of taxa treated in keys and the number of species for which gene sequence data is available. The gap is even greater if you include described taxa that can be recognized by morphological description but are not in a published key. No doubt a large database of gene sequence data will eventually be available for many taxa. However, it is highly unlikely that for most taxa, especially fossils and most museum specimens, there will ever be sequence data. The taxon-by-sequence database will always have vast gaps in both directions. Having sequence data of an

\footnotetext{
${ }^{2}$ Herbert et al. actually use the term "gender", we assume they mean biological sex.
} 
unidentifiable semaphoront is only useful if an identified or identifiable individual has already been sequenced-and sequenced for the same gene region-and there is an understanding of variation among individuals. These are serious restrictions. For very rare taxa, known only from non-DNA quality museum specimens, it will remain impossible to place a second specimen, even if the new specimen's entire genome is sequenced. For those groups lacking differential keys, someone will have to make an initial assessment of taxon boundaries to produce the names to be used. Therefore the dependence on morphological expertise will be little diminished while a great deal of money and effort will be shifted to molecular endeavors. The use of small segments of DNA for identification (inexplicably and incorrectly referred to as "microgenomic" by Hebert et al.) does nothing to alleviate this problem.

\footnotetext{
"...the use of keys often demands such a high level of expertise that misdiagnoses are common."
}

It is unclear what data are used to substantiate the authors claim that "misdiagnoses are common." We assume this is based on the authors' own experiences and does not necessarily reflect the situation in biological publications at large. The necessity that biologists learn details of their organisms to ensure correct identification can slow the rate of publication and may prevent or delay the proposal of broad hypotheses based on sequence data extracted from otherwise unknown entities. It is certainly harder, or at least more time consuming, to learn and implement the methods used in morphological identification than it is to use packaged DNA extraction and PCR kits. However, as we show, DNA-based identification as presented by Hebert et al. is flawed and will in many cases not lead to any greater confidence in identification, nor will it free us from the need to know and understand morphological systems. It will, however, abandon important tools needed by biologists of every stripe. With regard to developing expertise, there is a very straightforward fix. Train more personnel in morphological techniques; reinstate the teaching of systematics, taxonomy and morphological techniques as core courses. In part programs such as the NSF PEET (Partnerships for Enhancing Expertise in taxonomy (2003): http://www.nsf.gov/pubsys/ods/getpub.cfm?nsf00140) have already recognized and begun to rectify this problem.

\section{Problems of implementation: defining species}

Hebert et al. point out the greatest weakness of their methods.

\footnotetext{
"However, there is no simple formula that can predict the length of sequence that must be analyzed to ensure species diagnosis, because rates of molecular evolution vary between different segments of the genome and across taxa."
}

Despite this statement, and the actual inability of knowing when results are a misdiagnosis, the authors were not dissuaded from establishing arbitrary standards for the correct placement of test taxa or from using a single data source, undoubtedly subject to the wide range of divergence rates which they note as obvious.

As stated by Hebert et al. a preordained length of sequence may or may not yield enough information for species identification due to variance in rates of molecular evolution across groups. Moreover, even the selection of an ostensibly informative part of one mitochondrial gene is problematic. This is because the maternally inherited mitochondrial genome sorts independently from the nuclear genome (which contains the majority of the genetic information defining lineages). Therefore, a study based solely on limited mitochondrial data (without even the illumination provided by some morphological knowledge) might only reflect the inheritance pattern of the mitochondrial genome and not that of the individual as a whole, due to differences between species sorting and gene sorting (Avise, 2000). Across different phyla, reliance on just a part of the mitochondrial genome was shown to result in paraphyletic species associations (Hedin, 1997; Patton and Smith, 1994; Sperling and Harrison, 1994; Talbot and Shields, 1996), which would result in misleading species identifications for what are otherwise considered different monophyletic taxa. A recent study of Phyciodes butterflies demonstrated that using just the part of the mitochondrial genome recommended by Hebert et al. regularly fails to correctly identify an insect to species, especially when branch lengths are relatively short (Wahlberg et al., 2003). Even translating sequence into amino acids was problematic at deeper divergences and resulted in some family level mis-identifications (Wahlberg, 2003). It has already been established that the combination of a predetermined segment of sequence with an inheritance pattern that may not mirror the rest of the genome makes the use of a portion of mitochondrial DNA a poor choice as the sole source of data for species identifications. Hebert et al. do little to refute this evidence.

In addition to the practical problems mentioned above, there are philosophical problems with species delineations that are not addressed by Hebert et al. They assert that DNA barcoding will lead to a future where "the bounds of intraspecific diversity will be quantifiable, sibling species will be recognizable, taxonomic decisions will be objective and all life stages will be identifiable." All but the last of these claims demonstrate an apparent ignorance regarding modern theories of cladogenesis and speciation. Research in the field of speciation has indicated that there are a multitude of different biological and historical conditions that may or may not ultimately lead to lineage 
divergence or reticulation (Wilson, 1999; Wheeler and Meier, 2000). What defines "species" is an intractable debate that cannot be resolved satisfactorily using part of a single gene. No single process or pattern can define or identify all species, and no single character set can adequately track and therefore reliably recognize even most species. This is especially true for closely related species, where taxa are in the process of diverging or recently diverged and are frequently represented by incomplete genomic sorting (Avise, 2000).

\section{Conclusions}

One of the benefits of the mtDNA identification methods touted by Hebert et al. that we do not dispute is the possibly reliable identification of most specimens of insects to Order. However, given that most workers with even a crude background in entomology can successfully identify insects to order on sight, it would not be worth the expense and time of a sequencing experiment to do this routine task. This is essentially what Sperling (2003) noted: that barcode methods may work "in all except the kinds of identifications that matter most." In fact, these methods seem prone to failure except in cases with an extremely well developed background knowledge of the taxa to be sampled and an a priori understanding of sequence variation among populations and individuals.

From the perspective of a general philosophy regarding human discovery and knowledge of the natural world, a strictly molecular approach to inquiry would result in a sterile intellectual landscape. Patterns that humans perceive in nature are derived from an understanding of all types of data, particularly rich data types like morphology. These observed patterns are the source and raison d'être for the value we see in biodiversity. A holistic view of organisms incorporating phylogeny, functional morphology, behavior, ecology, etc., helps us to make informed conservation decisions. How would decisions be made in a world where our view of animals was restricted to clusters divided by an arbitrary difference in their COI sequence, say, 5\% divergent? It is hard to imagine any general theorem of biology emerging in such a limited system or that people in general would remain interested in biodiversity at all.

Hebert et al. claim that "a COI identification system" for species will be reliable, cost-effective and accessible. In fact this method fails in all but the latter claim. In terms of accessibility, the DNA data format does allow for concise and unambiguous transfer via the internet. However, as pointed out by Seberg et al. (2003), access will favor wealthier countries, further dividing nations with the most biodiversity from those nations with the most control over biodiversity resources. We have shown that the claim of reliability or even relatively greater reliability over morphologybased identifications is specious. Purported cost-effectiveness, even if the methods worked, is a hollow claim. Biological science would sacrifice by completely shifting resources and attention from whole organisms to a very small segment of the genome. Our ability to tap the legacy of morphological and natural history data would be lost, and this would greatly impede possibilities for future theoretical advances in our understanding of the world.

Clearly, DNA sequence data is an important and powerful part of taxonomy and systematics. Molecular data has an indisputable role in the analysis of biodiversity. However, DNA-based data should not be seen as a substitute for understanding and studying whole organisms when determining identities or systematic relationships. Specific cases demand the use of molecules if we are to address questions that defy resolution using other character systems. The notion that there is an inherent supremacy of DNA data vs. other types of character data for all taxonomic questions and circumstances is wrongheaded. A clear example of these mistaken notions was recently published by Scotland et al. (2003b). Such publications and specifically Hebert et al. $(2003 \mathrm{a}, \mathrm{b})$ demand a balanced response that considers the role of morphology in taxonomy more carefully and reveals the actual costs and products of technologically attractive alternatives (Scotland et al., 2003a). By pointing out some of the shortcomings of the methods employed by Hebert et al. (2003a) on a variety of levels, we hope to draw attention to the damage such solely molecular approaches and accompanying analyses might cause to the important endeavor of assessing and understanding global biodiversity.

\section{Acknowledgments}

We thank Q. Wheeler, Cornell University, A. Seago and J. Powell, University of California, Berkeley for comments. This paper is journal number 4670 in the College of Tropical Agriculture and Human Resources, The University of Hawaii.

\section{References}

Avise, J.C., 2000. Phylogeography: the History and Formation of Species. Harvard University press, Cambridge, MA.

Estabrook, G.F., 1978. Some concepts for the estimation of evolutionary relationships in systematic botany. Syst. Bot. 3, 146-158.

Estabrook, G.F., 1986. Evolutionary classification using convex phenetics. Syst. Zool. 35, 560-570.

Hebert, P.D.N., Cywinska, A., Ball, S.L., deWaard, J.R., 2003a. Biological identifications through DNA barcodes. Proc. R. Soc. Lond. Ser. B 270, 313-321. 
Hebert, P.D.N., Ratnasingham, S. and deWaard, J.R., 2003b. Barcoding animal life: cytochrome c oxidase subunit 1 divergences among closely related species. R. Soc. Lond. Ser. B Suppl. 270, S96-S99.

Hedin, M.C., 1997. Speciational history in a diverse clade of habitatspecialized spiders (Araneae: Nesticidae: Nesticus): inferences from geographic-based sampling. Evolution, 51, 1929-1945.

Kumar, S., Gadagkar, S., 2000. R. Efficiency of the neighbourjoining method in reconstructing deep and shallow evolutionary relationships in large phylogenies. J. Mol Evol. 51, 544-553.

Lipscomb, D., Platnick, N., Wheeler, Q., 2003. The intellectual content of taxonomy: a comment on DNA taxonomy. Trends Ecol. Evol. $18(2), 64-66$.

Novotny, V., Basset, Y., Miller, S.E., Weiblen, G.D., Bremer, B., Cizek, L., Drozd, P., 2002. Low host specificity of herbivorous insects in a tropical forest. Nature 416(6883), 841-844.

Patton, J.L., Smith, M.F., 1994. Paraphyly, polyphyly, and the nature of species boundaries in pocket gophers (genus Thomomys). Syst Biol. 43, 11-26.

Pennisi, E., 2003. Modernizing the tree of life. Science 300(5626) 1692-1697.

Schlichting, D., Pigliucci, M., 1998. Phenotypic Evolution: a Reaction Norm Perspective. Sinauer, Sunderland, MA

Scotland, R.W., Hughes, C., Bailey, D., Wortley, A., 2003a. The Big Machine and the much-maligned taxonomist. Syst. Biodiversity 1(2), 139-143.

Scotland, R.W., Olmstead, R.G., Bennett, J.R., 2003b. Phylogeny reconstruction: The role of morphology. Syst. Biol. 52(4), 539-548.

Seberg, O., Humphries, C.J., Knapp, S., Stevenson, D.W., Petersen, G., Scharff, N. and Andersen, N.M., 2003. Shortcuts in systematics? A commentary on DNA-based taxonomy. Trends Ecol Evol. 18(2), 63-65.

Sperling, F., 2003. DNA barcoding. Deux et machina. Newsl. Biol Surv. Can. (Terrestrial Arthropods), Opin. Page 22(1), <http:/ www.biology.ualberta.ca/bsc/news22 2/contents.htm >.
Sperling, F.A.H., Harrison, R.G., 1994. Mitochondrial DNA variation within and between species of the Papilio machaon group of swallowtail butterflies. Evolution 48, 408-422.

Stoeckle, M., 2003. Taxonomy. DNA, bar code life. Bioscience 53(9), $2-3$

Stoeckle, M., Janzen, D., Hallwachs, W., Hanken and Baker, J., 2003. Draft conference report. Taxonomy, DNA and the barcode of life. Meeting held at the Banbury Center, Cold Spring Harbor Laboratory, New York, NY, September 10-12, 2003. Sponsored by the Sloan Foundation. < http://phe.rockefeller.edu/BarcodeConference/docs/B2summary.doc $>$.

Talbot, S.L., Shields, G.F., 1996. Phylogeography of brown bears (Ursus arctos) of Alaska and paraphyly within the Ursidae. Mol. Phylogenetics Evol. 5, 477-494.

Tautz, D., Arctander, P., Minelli, A., Thomas, R.H., Vogler, A.P., 2002. DNA points the way ahead in taxonomy. Nature. 418(368), 479

Tautz, D., Arctander, P., Minelli, A., Thomas, R.H., Vogler, A.P., 2003. A plea for DNA taxonomy. Trends Ecol. Evol. 18(2), 71-74.

Wahlberg, N., 2003. The breaking of the sequencer: Phyciodes and the fallacy of DNA barcodes. Presentation, meeting of the Lepidopterist's society.

Wahlberg, N., Oliveira, R., Scott, J.A., 2003. Phylogenetic relationships of Phyciodes butterfly species (Lepidoptera: Nymphalidae): complex mtDNA variation and species delimitations. Syst. Entomol. 28, 257-273.

Wheeler, Q.D., Meier, R., 2000. Species Concepts and Phylogenetic Theory: A Debate. Columbia University Press, New York.

Whitfield, J., 2003. DNA barcodes catalogue animals. < http:// www.nature.com/nsu/nsu_pf/030512/030512-7.html > . Nature Science Update.

Wilson, R.A., 1999. Species: New Interdisciplinary Essays. MIT Press, Cambridge, MA. 